\title{
BMJ Open Effects of telephone-delivered lifestyle support on the development of diabetes in participants at high risk of type 2 diabetes: J-DOIT1, a pragmatic cluster randomised trial
}

Naoki Sakane, ${ }^{1}$ Kazuhiko Kotani, ${ }^{1,2}$ Kaoru Takahashi, ${ }^{1,3}$ Yoshiko Sano, ${ }^{1,4}$ Kokoro Tsuzaki, ${ }^{1}$ Kentaro Okazaki, ${ }^{5}$ Juichi Sato, ${ }^{6}$ Sadao Suzuki, ${ }^{7}$ Satoshi Morita, ${ }^{8}$ Yoshitake Oshima, ${ }^{9}$ Kazuo Izumi, ${ }^{10}$ Masayuki Kato, ${ }^{11}$ Naoki Ishizuka, ${ }^{12}$ Mitsuhiko Noda, ${ }^{11}$ Hideshi Kuzuya ${ }^{1,13}$

To cite: Sakane N, Kotani K, Takahashi K, et al. Effects of telephone-delivered lifestyle support on the development of diabetes in participants at high risk of type 2 diabetes: J-DOIT1, a pragmatic cluster randomised trial. BMJ Open 2015;5:e007316

doi:10.1136/bmjopen-2014007316

- Prepublication history for this paper is available online. To view these files please visit the journal online (http://dx.doi.org/10.1136/ bmjopen-2014-007316).

Received 27 November 2014 Revised 17 February 2015 Accepted 3 March 2015

CrossMark

For numbered affiliations see end of article.

Correspondence to

Dr Naoki Sakane;

nsakane@kyotolan.hosp.go.jp

\section{ABSTRACT}

Objectives: To examine the effects of telephonedelivered lifestyle coaching on preventing the development of type 2 diabetes mellitus (T2DM) in participants with impaired fasting glucose (IFG).

Design: Cluster randomised trial.

Setting: 40 groups from 17 healthcare divisions in Japan: companies (31), communities (6) and mixed settings (3).

Participants: Participants aged 20-65 years with fasting plasma glucose (FPG) of $5.6-6.9 \mathrm{mmol} / \mathrm{L}$ were invited from the 17 healthcare divisions.

Randomisation: The groups were then randomly assigned to an intervention or a control arm by independent statisticians according to a computergenerated list.

Intervention: The intervention arm received a 1-year telephone-delivered intervention provided by three private lifestyle support centres (at different frequencies: low-frequency (3 times), middle-frequency (6 times) and high-frequency (10 times) support calls). The intervention and control arms both received selfhelp devices such as a weight scale and pedometer.

Outcomes: Participants were followed up using data from annual health check-ups and a questionnaire regarding lifestyle. The primary outcome was the development of T2DM defined as FPG $\geq 7.0 \mathrm{mmol} / \mathrm{L}$, the diagnosis of diabetes, or use of an antidiabetic drug, confirmed by referring to medical cards.

Results: Of 14473 screened individuals, participants were enrolled in either the intervention $(n=1240)$ arm or control $(n=1367)$ arm. Overall, the HR for the development of T2DM in the intervention arm during 5.5 years was $1.00(95 \% \mathrm{Cl} 0.74$ to 1.34$)$. In the subanalysis, the HR was $0.59(95 \% \mathrm{Cl} 0.42$ to 0.83$)$ in the subgroup that received phone calls the most frequently, compared with the control arm. A limitation of the study includes a lack of blinding.

Conclusions: High-frequency telephone-delivered lifestyle support could effectively prevent T2DM in

\section{Strengths and limitations of this study}

Findings were retrieved from a well-designed cluster randomised controlled trial.

- This translational study included large nationwide samples, a wide range of ages and body mass index, and real-world settings.

- All the data for the study were from health check-up sites in companies and communities. We did not include any other biochemical examinations, such as the oral glucose tolerance test.

- For identifying impaired fasting glucose and diagnosing diabetes, we used a single test result of fasting plasma glucose determined at an annual health check-up. Because of poor reproducibility, we must be careful when interpreting a single test result. In addition, we may have missed diabetic and impaired glucose tolerance participants with normal fasting but elevated $2 \mathrm{~h}$ plasma glucose levels.

- A limitation of the study includes a lack of blinding.

participants with IFG in a primary healthcare setting, although low-frequency and middle-frequency phone calls did not.

Trial registration number: This trial has been registered with the University Hospital Medical Information Network (UMIN000000662).

\section{BACKGROUND}

Type 2 diabetes mellitus (T2DM) continues to be a major health issue in the 21st century. ${ }^{1}{ }^{2}$ There is an urgent need for effective strategies to contain this pandemic. The Finnish Diabetes Prevention Study (DPS $)^{3}$ and US Diabetes Prevention Program 
(DPP $)^{4}$ both found that intensive lifestyle intervention can prevent or delay the development of T2DM in highrisk populations. However, translating the findings of clinical research into real-world primary healthcare practice on a large scale remains to be achieved. ${ }^{5}$ In Japan, to contain increases in lifestyle-related diseases, including T2DM, health check-ups targeting metabolic syndrome (Tokutei Kenshin) were introduced in 2008 by the government. ${ }^{6}$ Through these health check-ups, a large number of high-risk participants for T2DM are identified every year. For those high-risk individuals, lifestyle interventions are implemented using a variety of modalities, including face-to-face individual or group sessions and non-face-to-face healthcare support. It is, however, questionable to what extent annual health check-ups contribute towards overcoming the pandemic of T2DM. ${ }^{7}$ There is a large gap between identifying high-risk participants and preventing diabetes in the real world. Given the limited resources for primary healthcare, cost-effectiveness is a key issue. Evidence-based effective and efficient prevention programmes that are easily accessible are needed. Telephone counselling support would make it possible to deliver lifestyle intervention widely, ${ }^{8-10}$ at low cost, yet in a personalised manner. If proven effective, it would be a promising tool for national strategies against T2DM. The Japan Diabetes Outcome Intervention Trial-1 (J-DOIT1) is a nationwide, pragmatic cluster randomised controlled trial. ${ }^{11}$ The aim of this study was to investigate whether goal-focused lifestyle coaching delivered by telephone can effectively prevent the development of T2DM in high-risk individuals in a primary healthcare setting. We also aimed to clarify the effectiveness of telephone programmes for lifestyle support by comparing three different protocols.

\section{METHODS}

\section{Research design}

The protocol, rationale and recruitment of the study participants are described elsewhere. ${ }^{12}$ A cluster randomised controlled trial was conducted at healthcare divisions in companies and communities that carry out health check-ups for employees and residents. Randomisation occurred at the level of branches of the healthcare division to avoid contamination between the intervention and control arms.

\section{Recruitment}

\section{Healthcare divisions}

By advertising on the internet or through direct contact, we invited healthcare divisions in communities and at worksites (companies) to participate in the study. The inclusion criteria for the divisions were: (1) health check-ups were conducted according to guidelines by the Health Promotion Law, (2) there were 2000 or more examinees annually, (3) the study group could be provided with health check-up data every year starting from
2006 and (4) lifestyle surveys could be conducted every year using a questionnaire prepared by the study team.

\section{Randomisation}

The branches (groups) of healthcare divisions recruited from communities and companies were the randomisation unit. The groups were then randomly assigned to an intervention or a control arm by independent statisticians according to a computer-generated list. The groups were notified of their allocation status before study participants were recruited.

\section{Participants}

Using the 2006 health check-up data, candidates who met the inclusion criteria were identified in each group. Inclusion criteria included an age of 20-65 years and impaired fasting glucose (IFG), defined as a fasting plasma glucose (FPG) concentration of $100-125 \mathrm{mg} / \mathrm{dL}$ $(5.6-6.9 \mathrm{mmol} / \mathrm{L})$. Exclusion criteria included diagnosed diabetes, a history of taking antidiabetic agents and glycated haemoglobin of $\geq 6.5 \% .{ }^{13}$ Women with a history of gestational diabetes could be enrolled. Physical or medical conditions that do not allow exercise, pregnancy or possible pregnancy, T1DM, liver cirrhosis or chronic viral hepatitis, and the use of a cardiac pacemaker, were also included in exclusion criteria. The recruitment of the participants started on 31 March 2007 and ended in March 2011.

\section{Lifestyle support centre}

We outsourced some parts of the study to three existing private companies (Tokio Marine \& Nichido Medical Service Co, Ltd, Tokyo, Japan; National Education Association, Inc, Visit Health Co, Ltd, Tokyo, Japan; and Meiji Yasuda System Technology Co, Ltd, Tokyo, Japan). They are all practising healthcare services. They participated in this study as lifestyle support centres, managing recruitment and enrolment of study participants and the lifestyle intervention.

\section{Intervention arm}

The goals for lifestyle change were set for each participant referring to the following four points: (1) habitual exercise (10000 steps or more per day or $60 \mathrm{~min}$ or more per week of accumulated moderate exercise) ${ }^{14} 15$ (2) achievement and maintenance of an appropriate body weight (a 5\% reduction in body weight in participants with a body mass index $(\mathrm{BMI}) \geq 25 \mathrm{~kg} / \mathrm{m}^{2}$ or a $3 \%$ reduction in participants with a BMI of $23.0-24.9 \mathrm{~kg}$ / $\mathrm{m}^{2}$ ), (3) habitual intake of dietary fibre (five or more servings of vegetables per day or $350 \mathrm{~g}$ or more of vegetables per day ${ }^{16}$ and (4) restrictions on alcohol intake (1 'go' $(180 \mathrm{~mL})$ or less per day in terms of Japanese sake: 1 'go' of Japanese sake contains $23 \mathrm{~g}$ of ethanol ${ }^{17}$ ). After setting goals, a weight scale (HBF-354 IT-2, Omron Healthcare Co, Ltd) and a pedometer (HJ-710 IT, Omron Healthcare Co, Ltd) with a storage function were provided. They could send accumulated data to the 
lifestyle support centres via a transmitter (DC-100, JMS Co, Ltd). The participants periodically received newsletters from the lifestyle support centres, which included health-related information and messages to regularly encourage them to undergo health check-ups. Telephone-delivered lifestyle support was given by healthcare providers (nurses, public health nurses and dieticians) over a 1-year period through one of the three lifestyle support centres. Participating groups were allocated to one of the three lifestyle support centres (designated as centre A, centre B and centre $\mathrm{C}$ ). Participants received lifestyle support calls 3 times from centre A, 6 times from centre $\mathrm{B}$ and 10 times from centre $\mathrm{C}$ over 1 year, with the length of each call being between 15 and $30 \mathrm{~min}$. Centres A and $\mathrm{C}$ also sent monthly advice sheets by mail. We held educational sessions on diabetes and its prevention for healthcare providers in each support centre and training sessions to improve their telephone counselling skills with motivational interviewing. The staff at each centre conducted the baseline assessment (lifestyle, motivation for lifestyle modifications, stage of change, health status and knowledge of diabetes) and set the personal action plan: (1) list of specific goals in behavioural terms, coaching in realistic and measurable goals to increase self-efficacy, (2) discussion of advantages and disadvantages of healthy behaviour changes, (3) identification of barriers to healthy behaviour changes and (4) discussion of problem-solving approaches to improve ability to address barriers. The participants were encouraged to measure their body weight and count footsteps every day, and send the accumulated data to the lifestyle support centres via a transmitter. The healthcare provider monitored progress towards the participant's goals regularly and gave advice by phone or mail.

\section{Control arm}

After setting the goals, a weight scale and pedometer were also provided for participants in the control arm. Participants in the control arm periodically received newsletters on diabetes and diabetes prevention but did not receive telephone-delivered intervention. Participants were encouraged, through newsletters, to undergo annual health check-ups.

\section{Outcome measures}

Participants were followed up annually using the data from an annual health check-up, and a questionnaire regarding health and lifestyle. The primary outcome was the development of diabetes in participants whose FPG concentration was $5.6-6.9 \mathrm{mmol} / \mathrm{L}(100-125 \mathrm{mg} / \mathrm{dL})$ at baseline. The development of T2DM was defined as: (1) a rise in FPG to a level $\geq 7.0 \mathrm{mmol} / \mathrm{L}(126 \mathrm{mg} / \mathrm{dL})$ as revealed in the annual follow-up health check-ups and (2) a diagnosis of diabetes or use of antidiabetic drugs as reported in the annual questionnaire with confirmation by referring to medical records. Overweight (23.0$24.9)$ and obesity $(\geq 25)$ were defined based on BMI $\left(\mathrm{kg} / \mathrm{m}^{2}\right)$ using the WHO Western Pacific Regional Office criteria. ${ }^{18}$ Other outcomes included a change in body weight, success of achieving goals, and adherence to and satisfaction with telephone intervention. Programme participation was evaluated by the number of responses to phone calls (percentage). Good adherence was defined as $>80 \%$. Total and telephone-related costs were calculated. Satisfaction of participants with the support service was assessed based on six items (overall; provision of knowledge on diabetes and diabetes prevention; dietary support; exercise support; wanting to continue to receive telephone support in the future; recommending the telephone support service to other people such as family members and friends). The responses were rated on a six-point Likert scale (ranging from 1 (strongly disagree) to 6 (strongly agree)). Dropout cases in the present study include: (1) participants who have not undergone an annual health check-up after enrolment and (2) participants who have lost contact with the study team.

\section{Analysis and power}

Masking of treatments was not possible because implementation of the intervention was obvious. Data analysts were not blinded after the database was locked for final analysis. The analyses were conducted on an intention-to-treat basis, using Statistical Package for SAS V.9.3 (SAS Inc, Cary, North Carolina, USA) and Statistical Package for Social Science software V.20.0 (IBM SPSS Inc, Chicago, Illinois, USA). Survival curves for the development of diabetes were estimated by the Kaplan-Meier method. The log-rank test was also used. We took into account the clustering effect in the main outcome analysis and subanalysis using the LWA model (Lee, Wei and Amato) ${ }^{20}$ Cox regression analysis was used to calculate the HR and $95 \%$ CI. The subanalyses were performed within each lifestyle support centre because each lifestyle support centre was in charge of each intervention and control arm. Also, Cox regression analysis was adjusted for baseline FPG concentration. Values are presented as the mean \pm SD. Student $t$ test (or the Mann-Whitney U test according to the frequency distribution of the variable) was used to compare the means (or distributions) of the two study arms for continuous variables. A $\chi^{2}$ test was used to compare proportions for categorical variables. Missing data on other outcomes were handled using the last observation carried forward imputation technique. The $p$ value $<0.05$ was considered significant. Based on the available prospective data from the Japanese population, the yearly incidence of diabetes among the high-risk group varies between $2 \%$ and $7 \% .^{21}$ When calculated on the assumption that the annual incidence of diabetes is $4 \%$ in the control arm and the intervention reduces the incidence by $50 \%$, the number is 1100 with an $\alpha$ of $5 \%$ and a power of $90 \%$. When the intra-cluster correlation coefficient (ICC) and cluster size (number of individuals in each cluster) are assumed to be 0.02 and 60 , participants and the number of clusters 
Table 1 Baseline characteristics of control and intervention groups and participants

\begin{tabular}{|c|c|c|}
\hline Variables & Intervention arm (20 groups, $n=1240$ ) & Control arm (20 groups, $n=1367$ ) \\
\hline \multicolumn{3}{|c|}{ Group factors at baseline } \\
\hline \multicolumn{3}{|c|}{ Settings (total number of participants) } \\
\hline Company setting & 16 groups $(n=1121)$ & 15 groups $(n=1197)$ \\
\hline Community setting & 3 groups $(n=94)$ & 3 groups $(n=92)$ \\
\hline Mixed sitting & 1 group $(n=25)$ & 2 groups $(n=78)$ \\
\hline \multicolumn{3}{|l|}{ Participants factors at baseline } \\
\hline Age, year & $48.9(7.8)$ & $48.9(7.5)$ \\
\hline Male, \% & 82.5 & 84.1 \\
\hline Body mass index, $\mathrm{kg} / \mathrm{m}^{2}$ & $24.4(3.2)$ & $24.3(3.1)$ \\
\hline \multicolumn{3}{|l|}{ Lifestyle goals } \\
\hline Habitual exercise* & 51.7 & 52.4 \\
\hline Dietary fibre intake $†$ & 0.0 & 0.2 \\
\hline Restrictions on alcohol intake & 42.7 & 40.7 \\
\hline
\end{tabular}

will be 2398 and 40, respectively. Assuming that the dropout rate is $30 \%, 3426$ participants are needed.

\section{RESULTS}

\section{Participant enrolment and baseline characteristics}

Forty-three groups, formed from 17 healthcare divisions in companies or in communities across the country, were randomly assigned to a control arm (21 groups) or an intervention arm (22 groups) between March 2007 and February 2008. Approximately 230000 individuals (male, 85\%) underwent health check-ups in those 43 groups in 2006. Two groups in the intervention arm and one group in the control arm were excluded because FPG levels were not available at baseline. Among the groups, 14473 participants (7494 in the control and
Figure 1 CONSORT flow diagram of Japan Diabetes Outcome Intervention Trial-1 (J-DOIT1).

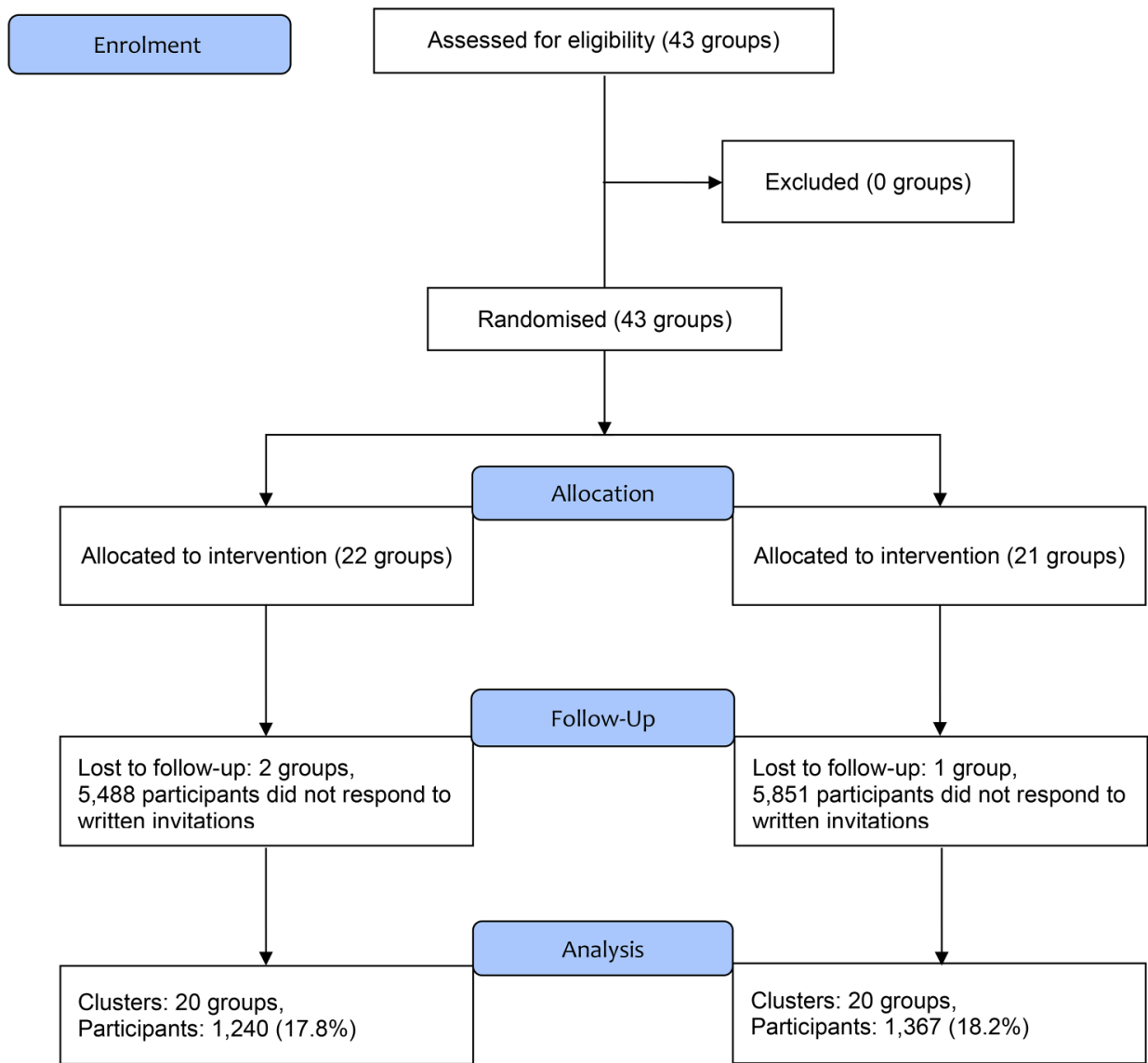


6979 in the intervention arm) received an invitation letter to participate in the study. Finally, 2607 participants were enrolled, with 1240 in the intervention and 1367 in the control arm (table 1) across the country (eg, Hokkaido, Tohoku, Kanto, Chubu, Kansai, Chugoku, Shikoku, Kyusyu and Okinawa ). The overall consent rate was approximately $18 \%$ with no difference between the study arms (figure 1). The median age, BMI and FPG level of the study participants were 49 years, $24.3 \mathrm{~kg} / \mathrm{m}^{2}$ and $5.9 \mathrm{mmol} / \mathrm{L}$, respectively, and $83.4 \%$ were men. There was no difference in the proportion of lifestyle goals (eg, dietary fibre intake and alcohol intake) at baseline between arms. Also, there was no difference in the proportion of lifestyle goals at baseline between arms within each centre. The dropout rate during the study was $17.5 \%$ for the intervention arm and $20.9 \%$ for the control arm.

\section{Primary outcomes}

During a median follow-up period of 4.2 years, T2DM developed in 115 participants in the intervention arm $(9.3 \%)$ and 132 participants in the control arm (9.7\%). Overall, the HR for the development of T2DM in the intervention arm during 5.5 years was 1.00 (95\% CI 0.74 to 1.34). In the subanalysis of the three lifestyle support centres, the HR was significantly reduced to 0.59 (95\% CI 0.42 to $0.83 ; \mathrm{p}=0.02$ ) for centre $\mathrm{C}$, which provided 10 telephone calls, while no beneficial effects on the incidence were found for centres $\mathrm{A}$ and $\mathrm{B}$, which made telephone calls less frequently. The HR was 1.41 (95\% CI 0.85 to $2.32 ; \mathrm{p}=0.18)$ for centre $\mathrm{A}$ and 1.19 (95\% CI 0.77 to $1.84 ; \mathrm{p}=0.38$ ) for centre $\mathrm{B}$ (figure 2 , table 2 ).

Overall, the baseline FPG adjusted for HR was 0.97 (95\% CI 0.69 to $1.38 ; \mathrm{p}=0.878$ ). The baseline FPG adjusted HR was 0.56 (95\% CI 0.34 to $0.94 ; \mathrm{p}=0.028$ ) for centre C, 1.55 (95\% CI 0.82 to 2.93; p0.176) for centre $\mathrm{A}$ and 1.12 (95\% CI 0.72 to $1.74 ; \mathrm{p}=0.617)$ for centre B.

\section{Other outcomes}

The decrease in body weight after 1 year intervention and at the end of the trial was modest, but significantly greater in the intervention arm than in the control arm (table 3). After 1 year intervention and at the end of the trial, the percentages of the participants who met the goal of 'weight reduction', 'dietary fibre intake' and 'restrictions on alcohol intake', were significantly higher in the intervention arm compared with the control arm. On the contrary, as to 'habitual exercise', no difference was found between the arms. When comparing each centre separately, centre $\mathrm{C}$, which provided highfrequency support calls, showed the most beneficial results. In centre $\mathrm{C}$, the proportion of participants within the normal range (FPG $<5.5 \mathrm{mmol} / \mathrm{L}$ ) was significantly higher in the intervention than control arm after 1 year intervention (table 3 ).

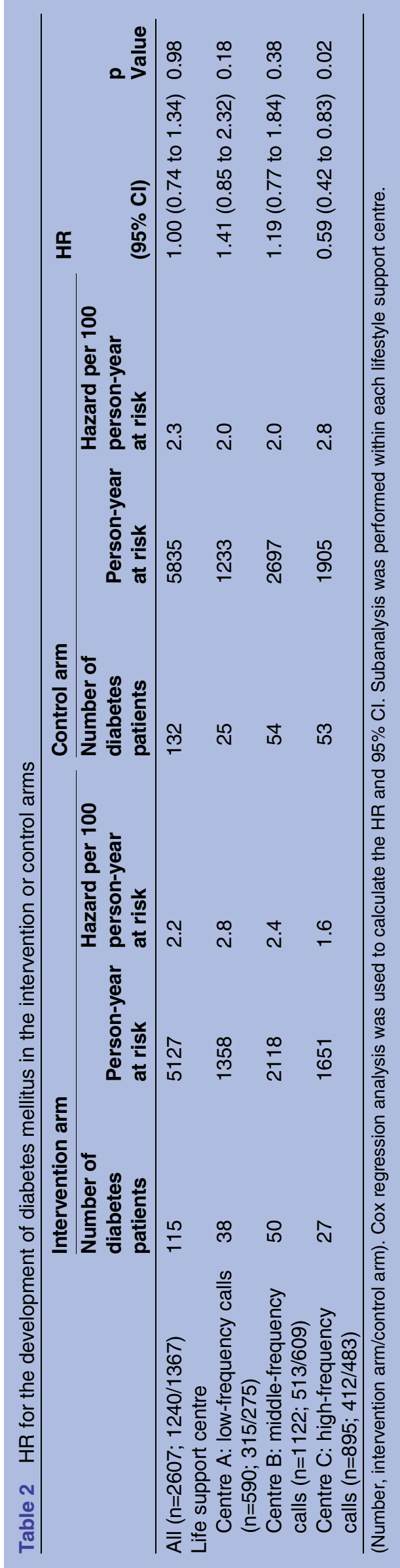




\section{Adherence and satisfaction}

The mean number of responses to the calls during the 1 year period was $2.8 \pm 0.6$ (range $1-3$ ) in centre A, 5.2 \pm 1.9 (range 1-6) in centre $B$ and $8.2 \pm 3.5$ (range 1-13) in centre C. The rates of good adherence were $91.4 \%$, $82.7 \%$ and $81.1 \%$, for each centre, respectively. Satisfaction of the participants with the support service was assessed in terms of six items. The score was slightly but significantly higher for each item in centre C (satisfaction score, evaluated on a six-point Likert scale on a maximum scale of 36 , centre A: 24.2 of 36 , centre B: 24.0 of 36 , centre C: 27.0 of 36 ).

\section{Adverse events}

There was no difference in the incidence of adverse events between the intervention and control arms $(1.9 \%$ vs $1.8 \%$, respectively, $\mathrm{p}=0.73)$. The numbers of participants who developed cancer, musculoskeletal system problems, ischaemic heart disease, stroke and other adverse events were 5, 6, 1, 3 and 9 in the intervention arm, and 8, 3, 2, 2 and 9 in the control arm, respectively. Four cases of musculoskeletal system problems in the intervention arm and one case of musculoskeletal problems in the control arm might have been related to the study treatment, while other adverse events were not. There
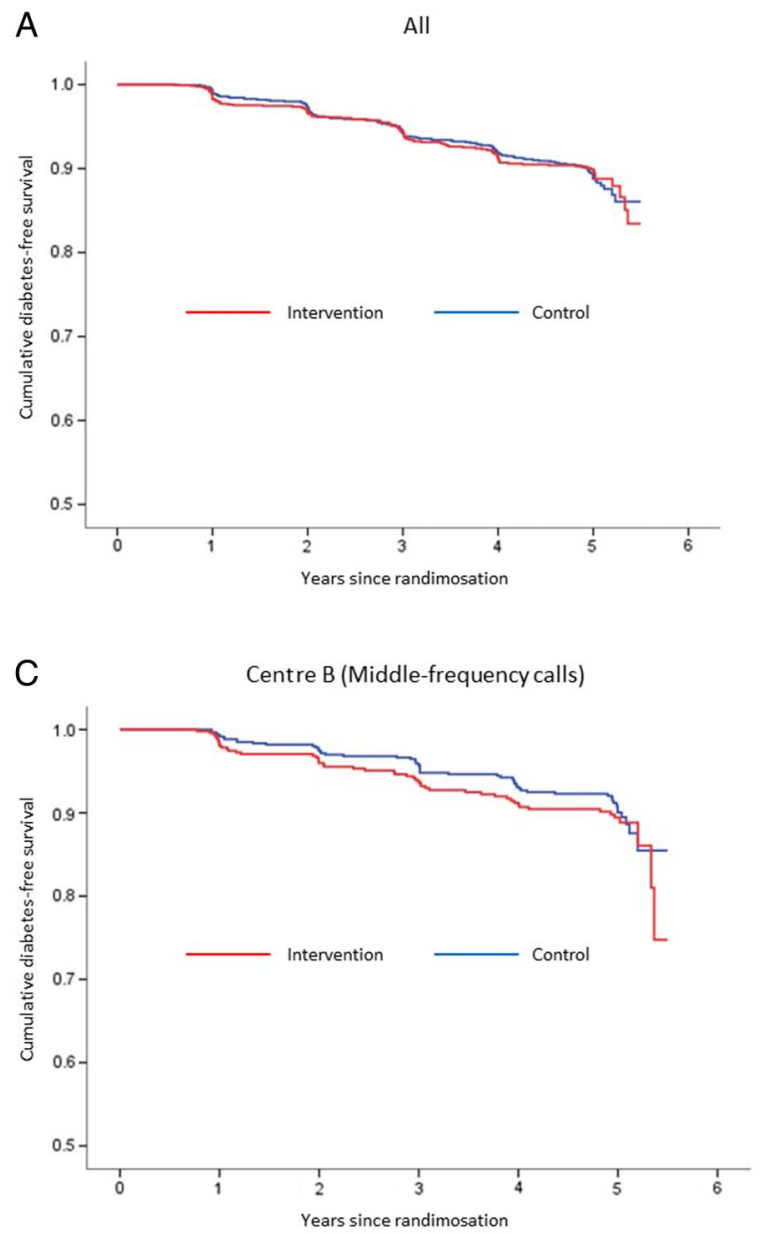

was no difference in the incidence of adverse events among the three centres.

\section{DISCUSSION}

It has become mandatory for all Japanese adults to undergo health check-ups at least once a year in workplaces or communities. Health check-ups are therefore becoming a routine part of healthcare. Through this health check-up, we recruited a large number of participants at high risk for T2DM from healthcare divisions across Japan. The present study was thus expected to generate much evidence regarding antidiabetes strategies. We included not only overweight and obese participants, but also participants with a BMI of $<23 \mathrm{~kg} / \mathrm{m}^{2}$. Therefore, the BMI ranged widely from $<18.5$ to $>30 \mathrm{~kg} /$ $\mathrm{m}^{2}$ in our study participants, with an average value of 24.3. Compared with Western populations, obesity is less common in Japanese in general. ${ }^{22}$ It has also been reported that about $25 \%$ of participants with impaired glucose tolerance (IGT) have normal or even underweight categories of BMI. ${ }^{23}$ It seems that the relationship between BMI and the risk of diabetes is not straightforward in Japanese populations. Thus, we did not set the eligibility criteria in terms of BMI. It would be of interest

B Center A (Low-frequencycalls)

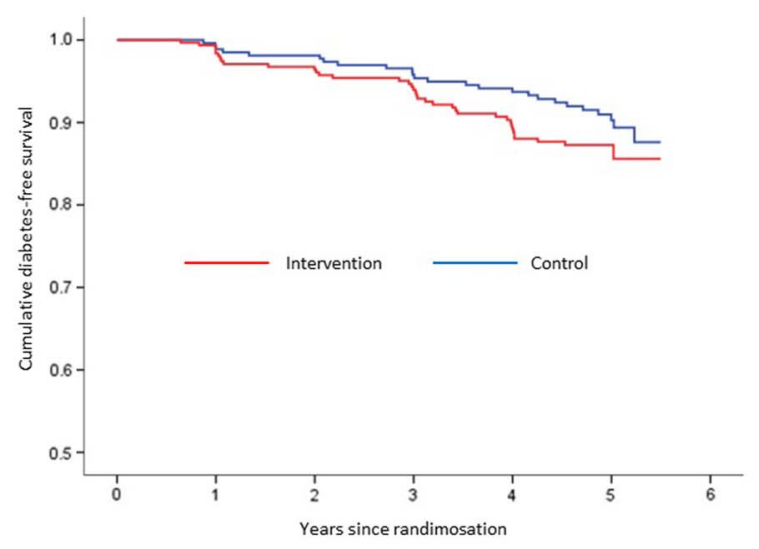

D

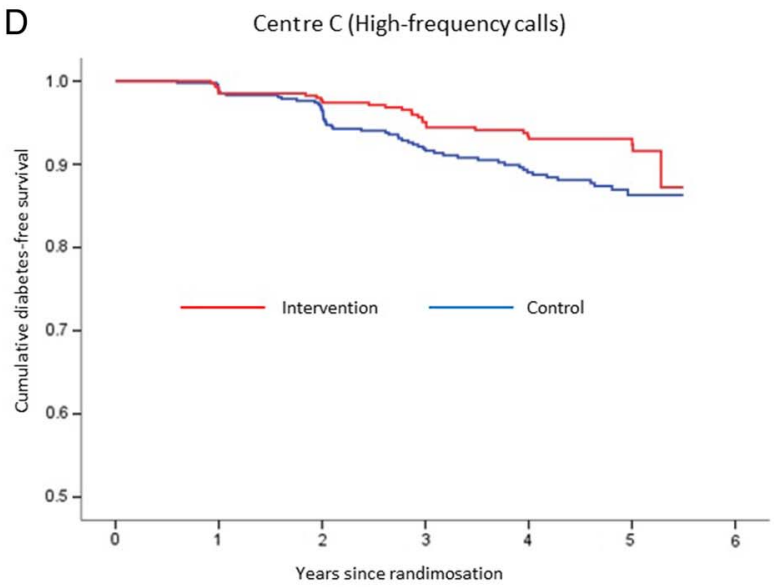

Figure 2 Kaplan-Meier plot for developing diabetes among all, low-frequency, middle-frequency and high-frequency calls. 
Table 3 Changes of parameters and success in achieving goals after 1 year intervention and trial end in the intervention or control arms

\begin{tabular}{|c|c|c|c|c|c|c|}
\hline & \multirow[b]{2}{*}{$\begin{array}{l}\text { Body } \\
\text { weight, kg }\end{array}$} & \multicolumn{4}{|c|}{ Lifestyle goals } & \multirow[b]{2}{*}{$\begin{array}{l}\text { FPG } \\
<5.5 \mathrm{mmol} / \mathrm{L}\end{array}$} \\
\hline & & $\begin{array}{l}\text { Habitual } \\
\text { exercise† }\end{array}$ & $\begin{array}{l}\text { Weight } \\
\text { reduction‡ }\end{array}$ & $\begin{array}{l}\text { Dietary fibre } \\
\text { intake§ }\end{array}$ & $\begin{array}{l}\text { Restriction on } \\
\text { alcohol intakeๆ }\end{array}$ & \\
\hline \multicolumn{7}{|l|}{ All } \\
\hline \multicolumn{7}{|l|}{ Intervention arm } \\
\hline 1 year after intervention & $-1.8(3.6)^{\star}$ & $71.6^{*}$ & $56.2^{*}$ & $5.6^{*}$ & $47.2^{*}$ & 42.1 \\
\hline End of trial & $-1.1(3.4)^{*}$ & 60.7 & $41.1^{*}$ & $12.3^{*}$ & 49.0 & 32.5 \\
\hline \multicolumn{7}{|l|}{ Control arm } \\
\hline 1 year after intervention & $-1.0(3.4)$ & 66.9 & 51.7 & 0.5 & 41.6 & 38.5 \\
\hline End of trial & $-0.6(3.6)$ & 59.2 & 36.1 & 6.0 & 45.6 & 32.4 \\
\hline \multicolumn{7}{|l|}{ Centre A } \\
\hline \multicolumn{7}{|l|}{ Intervention arm } \\
\hline 1 year after intervention & $-1.8(3.2)^{\star}$ & 74.8 & 58.8 & 2.2 & 40.3 & 31.0 \\
\hline End of trial & $-1.2(3.1)^{\star}$ & 60.0 & 41.6 & 8.6 & 41.3 & 26.8 \\
\hline \multicolumn{7}{|l|}{ Control arm } \\
\hline 1 year after intervention & $-1.1(3.3)$ & 69.8 & 56.2 & 0.5 & 35.7 & 27.2 \\
\hline End of trial & $-0.4(3.5)$ & 60.7 & 37.5 & 6.2 & 41.1 & 20.5 \\
\hline \multicolumn{7}{|l|}{ Centre B } \\
\hline \multicolumn{7}{|l|}{ Intervention arm } \\
\hline 1 year after intervention & $-1.6(3.8)^{\star}$ & 72.3 & 50.0 & $2.1^{*}$ & $44.9^{*}$ & 43.9 \\
\hline End of trial & $-0.9(3.4)$ & 62.0 & 37.8 & $7.0^{*}$ & 46.8 & 33.1 \\
\hline \multicolumn{7}{|l|}{ Control arm } \\
\hline 1 year after intervention & $-0.9(3.3)$ & 66.8 & 48.7 & 0.5 & 37.6 & 43.9 \\
\hline End of trial & $-0.6(3.4)$ & 59.3 & 33.8 & 4.3 & 43.2 & 37.2 \\
\hline \multicolumn{7}{|l|}{ Centre C } \\
\hline \multicolumn{7}{|l|}{ Intervention arm } \\
\hline 1 year after intervention & $-2.0(3.6)^{\star}$ & 68.0 & $61.7^{\star}$ & $12.4^{\star}$ & 55.8 & $49.4^{*}$ \\
\hline End of trial & $-1.3(3.5)^{\star}$ & 59.7 & $44.9^{*}$ & $21.8^{*}$ & 57.5 & 36.5 \\
\hline \multicolumn{7}{|l|}{ Control arm } \\
\hline 1 year after intervention & $-0.9(3.6)$ & 65.4 & 52.9 & 0.6 & 50.3 & 38.0 \\
\hline End of trial & $-0.6(3.8)$ & 58.2 & 38.3 & 8.1 & 51.1 & 33.1 \\
\hline \multicolumn{7}{|c|}{ 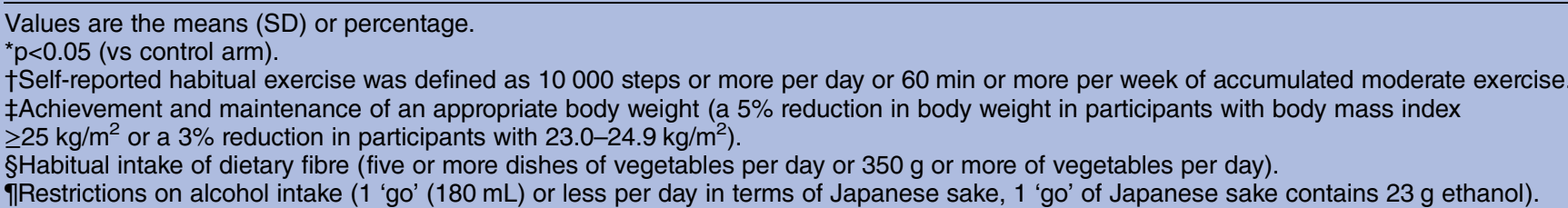 } \\
\hline
\end{tabular}

to study the incidence of diabetes and assess what strategies are effective to prevent the development of diabetes in those with a lower BMI.

All available data for the study were from health check-up sites in companies and communities. We did not include any other biochemical examinations, such as the oral glucose tolerance test. With an expanding population of patients with lifestyle-related diseases, healthcare businesses have grown and now play significant roles in combating such diseases. Therefore, to prevent T2DM in a real-world setting, we outsourced the implementation of lifestyle intervention to private healthcare businesses. Because of the large sample size, we employed three companies as lifestyle support centres in this study. The telephone-delivered lifestyle support was provided over a 1 year period by one of the three lifestyle support centres. Each company used its own intervention schedule approved by the study group.
The major difference in the protocol among the three centres was in the number of contacts by telephone.

\section{Main findings}

Overall, the study could not prove the effectiveness of a 1-year telephone-delivered lifestyle intervention programme to reduce the development of T2DM in participants with IFG. Changes in lifestyle by the 1 year intervention were modest. A small but significant amount of weight reduction was achieved in the intervention arm after the 1 year intervention and at the end of the trial. The proportions of participants who achieved and maintained an appropriate body weight, who achieved the goal of vegetable intake and who achieved the goal of alcohol intake, were all slightly higher in the intervention compared with the control arm. In this study, the control arm received a weight scale and a pedometer with a storage function, just as 
the intervention arm did. This was done to minimise the potential for the greater attrition of participants in the control arm. Thus, it seems likely that the use of these devices markedly contributed to the present findings on the lifestyle changes and incidence of diabetes. Therefore, to obtain additional interventional effects, intensive intervention would be needed. Consistent with this speculation, subanalysis of the three lifestyle support centres revealed that the HR was significantly reduced to 0.59 with centre $\mathrm{C}$, which provided 10 telephone calls, while no beneficial effects on the incidence were found with centres $\mathrm{A}$ and $\mathrm{B}$, which made telephone calls less frequently. From these findings, it would be reasonable to conclude that remote intervention delivered by telephone, if provided frequently, could be a beneficial measure for preventing the development of T2DM on a large scale. These findings are consistent with a previous study in obese patients with metabolic syndrome involving frequent telephone interventions (16-session core curriculum in year 1, 12-session continued telephone contact in year 2 plus telephone coaching sessions). ${ }^{24}$ Thus, we could propose that lifestyle support by telephone given monthly for 1 year might be a standard for effective intervention.

\section{Strengths and limitations}

The strengths of this translational study are that it included large nationwide samples, with wide range of ages and BMI, and was conducted in real-world settings. We could follow-up the study participants for 3 years after the intervention. Much evidence has been generated to contain ongoing T2DM, endemic in our country; several real-world translational studies based on intensive diabetes programmes have thus far provided important information necessary to reduce costs and increase accessibility. ${ }^{25-27}$ However, the main outcome of many of those studies was weight reduction, not development of diabetes. ${ }^{28}$

The study also had several potential limitations. First, all data were from health check-up sites in companies and communities. We did not include any other biochemical examinations, such as the oral glucose tolerance test. For identifying IFG and diagnosing diabetes, we used a single test result of FPG determined at an annual health check-up. Because of poor reproducibility, we must be careful when interpreting a single test result. ${ }^{29}$ In addition, we may have missed diabetic and IGT participants with normal fasting but elevated $2 \mathrm{~h}$ plasma glucose levels. ${ }^{30}$ Second, lifestyle data were based on self-reported questionnaires. Finally, the participants were predominantly from workplaces and we did not succeed in recruiting more participants from communities. Since men outnumber women in many workplaces in Japan, the study population was predominantly male. This bias may limit the generalisability of our results.

In summary, the present findings indicate that remote intervention delivered by telephone, if it is provided frequently, could be a beneficial measure in preventing the development of T2DM in a real-world primary care setting. Telephone-delivered lifestyle support by healthcare providers implemented monthly for 1 year might be a standard method for effective intervention.

\section{Author affiliations}

${ }^{1}$ Division of Preventive Medicine, Clinical Research Institute, National Hospital Organization Kyoto Medical Center, Kyoto, Japan

${ }^{2}$ Division of Community and Family Medicine, Juichi Medical University, Shimotsuke, Japan

${ }^{3}$ Hyogo Health Service Association, Kobe, Japan

${ }^{4}$ Kanagawa University of Human Services Faculty of Health \& Social Services School of Nutrition \& Dietetics, Yokosuka, Japan

${ }^{5}$ Department of Development for Community-oriented Healthcare System, Nagoya University Graduate School of Medicine, Nagoya, Japan

${ }^{6}$ Department of General Medicine/Family and Community Medicine, Nagoya

University Graduate School of Medicine, Nagoya, Japan

${ }^{7}$ Department of Public Health, Nagoya City University Graduate School of

Medical Sciences, Nagoya, Japan

${ }^{8}$ Department of Biomedical Statistics and Bioinformatics, Kyoto University Graduate School of Medicine, Kyoto, Japan

${ }^{9}$ University of Marketing and Distribution Sciences, Kobe, Japan

${ }^{10}$ Department of Diabetes and Metabolic Medicine, National Center for Global

Health and Medicine, Tokyo, Japan

${ }^{11}$ Department of Diabetes Research, Diabetes Research Center, National

Center for Global Health and Medicine, Tokyo, Japan

${ }^{12}$ Clinical trial department, Cancer Institute hospital, Tokyo, Japan

${ }^{13}$ Takeda Hospital, Kyoto, Japan

Acknowledgements The investigators gratefully acknowledge the health check-up divisions of the following companies and communities: Central Japan Railway Company, CO-OP Net, CSK Health Insurance Association, East Japan Railway Company, Hankyu Electric Railway Health Insurance Association, Health and Welfare Center (Atami), Hitachi Metals Health Insurance Society, Hitachi Transport System Health Insurance Association, JFE Steel Corporation, JTB Management Service Corp, Kakogawa General Health Care Center, Koga Health Examination Center, Meidensha Health Insurance Association, Mitsui Life Insurance Company Ltd, Tokyo Electric Power Company, SHARP Health Insurance Association and the Ube group (Ube City Office, Sanyo Onoda City Office, Tanabe Yamaguchi Pharma Factory Ltd, NISSAN Chemical Industries, Ltd, Nippon Kayaku Co, Ltd, and Sanyo Onoda City Health Center).

Contributors HK was involved in all aspects of the study. KI, MK, NI and MN designed the study, and prepared the protocol. NS, KK, YS, KT and KO were involved in drafting the manuscript. KT, JS, SS and SM participated in statistical analysis. All the authors have read and approved the final version of the manuscript.

Funding This study was funded by a Health and Labour Sciences Research Grant (Strategic Outcomes Research Program for Research on Diabetes and Comprehensive Research on Diabetes/Cardiovascular and Life-Style Related Diseases) from the Ministry of Health, Labour and Welfare, of Japan.

Competing interests None declared.

Patient consent Obtained.

Ethics approval This study was approved by the Ethical Committee of the Japan Foundation for the Promotion of International Medical Research Cooperation (Tokyo, Japan).

Provenance and peer review Not commissioned; externally peer reviewed.

Data sharing statement No additional data are available.

Open Access This is an Open Access article distributed in accordance with the Creative Commons Attribution Non Commercial (CC BY-NC 4.0) license, which permits others to distribute, remix, adapt, build upon this work noncommercially, and license their derivative works on different terms, provided the original work is properly cited and the use is non-commercial. See: http:// creativecommons.org/licenses/by-nc/4.0/ 


\section{REFERENCES}

1. Danaei G, Finucane MM, Lu Y, et al; Global Burden of Metabolic Risk Factors of Chronic Diseases Collaborating Group (Blood Glucose). National, regional, and global trends in fasting plasma glucose and diabetes prevalence since 1980: systematic analysis of health examination surveys and epidemiological studies with 370 country-years and 2.7 million participants. Lancet 2011;378:31-40.

2. Neville SE, Boye KS, Montgomery WS, et al. Diabetes in Japan: a review of disease burden and approaches to treatment. Diabetes Metab Res Rev 2009;25:705-16.

3. Tuomilehto J, Lindström J, Eriksson JG, et al; Finnish Diabetes Prevention Study Group. Prevention of type 2 diabetes mellitus by changes in lifestyle among subjects with impaired glucose tolerance. N Engl J Med 2001;344:1343-50.

4. Diabetes Prevention Program Research Group. Reduction in the incidence of type 2 diabetes with lifestyle intervention or metformin. N Engl J Med 2002;346:393-403.

5. Cardona-Morrell M, Rychetnik L, Morrell SL, et al. Reduction of diabetes risk in routine clinical practice: are physical activity and nutrition interventions feasible and are the outcomes from reference trials replicable? A systematic review and meta-analysis. BMC Public Health 2010;10:653.

6. Nakashima N, Kobayashi K, Inoguchi T, et al. A Japanese model of disease management. Stud Health Technol Inform 2007;129(Pt 2):1174-8.

7. Fukuda Y. Quasi-experimental study of effects of "healthcare advice with particular focus on metabolic syndrome". Nihon Eiseigaku Zasshi 2011;66:736-40.

8. Blackberry ID, Furler JS, Best JD, et al. Effectiveness of general practice based, practice nurse led telephone coaching on glycaemic control of type 2 diabetes: the Patient Engagement And Coaching for Health (PEACH) pragmatic cluster randomised controlled trial. BMJ 2013;347:f5272.

9. Appel LJ, Clark JM, Yeh HC, et al. Comparative effectiveness of weight-loss interventions in clinical practice. $N$ Engl J Med 2011;24:1959-68.

10. Digenio AG, Mancuso JP, Gerber RA, et al. Comparison of methods for delivering a lifestyle modification program for obese patients: a randomized trial. Ann Intern Med 2009;150:255-62.

11. Yazaki Y, Kadowaki T. Combating diabetes and obesity in Japan. Nat Med 2006;12:73-4.

12. Sakane N, Kotani K, Takahashi K, et al. Japan Diabetes Outcome Intervention Trial-1(J-DOIT1), a nationwide cluster randomized trial of type 2 diabetes prevention by telephone-delivered lifestyle support for high-risk subjects detected at health checkups: rationale, design, and recruitment. BMC Public Health 2013;13:81.

13. Kuzuya T, Nakagawa S, Satoh J, et al; Committee of the Japan Diabetes Society on the diagnostic criteria of diabetes mellitus. Report of the Committee on the classification and diagnostic criteria of diabetes mellitus. Diabetes Res Clin Pract 2002;55:65-85.

14. Tudor-Locke C, Hatano Y, Pangrazi RP, et al. How many steps/day are enough? For adults. Int J Behav Nutr Phys Act 2011;8:79.
15. Saito T, Watanabe M, Nishida J, et al. Lifestyle modification and prevention of type 2 diabetes in overweight Japanese with impaired fasting glucose levels: a randomized controlled trial. Arch Intern Med 2011;171:1352-60.

16. Hall JN, Moore S, Harper SB, et al. Global variability in fruit and vegetable consumption. Am J Prev Med 2009;36:402-9.

17. Lin $\mathrm{Y}$, Kikuchi S, Tamakoshi A, et al. Alcohol consumption and mortality among middle-aged and elderly Japanese men and women. Ann Epidemiol 2005;15:590-7.

18. Anuurad E, Shiwaku K, Nogi A, et al. The new BMI criteria for Asians by the regional office for the western pacific region of $\mathrm{WHO}$ are suitable for screening of overweight to prevent metabolic syndrome in elder Japanese workers. J Occup Health 2003;45:335-43.

19. Pan WH, Yeh WT. How to define obesity? Evidence-based multiple action points for public awareness, screening, and treatment: an extension of Asian-Pacific recommendations. Asia Pac J Clin Nutr 2008;17:370-4.

20. Mieno MN, Yamaguchi T, Ohashi Y. Alternative statistical methods for estimating efficacy of interferon beta- $1 \mathrm{~b}$ for multiple sclerosis clinical trials. BMC Med Res Methodol 2011;11:80.

21. Ito C, Maeda R, Nakamura K, et al. Prediction of diabetes mellitus (NIDDM). Diabetes Res Clin Pract 1996;34:S7-11.

22. Funatogawa I, Funatogawa T, Nakao M, et al. Changes in body mass index by birth cohort in Japanese adults: results from the National Nutrition Survey of Japan 1956-2005. Int J Epidemiol 2009;38:83-92.

23. Sakane N, Sato J, Tsushita K, et al. Prevention of type 2 diabetes in a primary healthcare setting: three-year results of lifestyle intervention in Japanese subjects with impaired glucose tolerance. BMC Public Health 2011;11:40.

24. Weinstock RS, Trief PM, Cibula D, et al. Weight loss success in metabolic syndrome by telephone interventions: results from the SHINE study. J Gen Intern Med 2013;28:1620-8.

25. Ma J, Yank V, Xiao L, et al. Translating the Diabetes Prevention Program lifestyle intervention for weight loss into primary care: a randomized trial. JAMA Intern Med 2013;173:113-21.

26. Hesselink AE, Bilo HJ, Jonkers R, et al. A cluster-randomized controlled trial to study the effectiveness of a protocol-based lifestyle program to prevent type 2 diabetes in people with impaired fasting glucose. BMC Fam Pract 2013;14:184

27. Sanchez A, Silvestre C, Sauto R, et al. Feasibility and effectiveness of the implementation of a primary prevention programme for type 2 diabetes in routine primary care practice: a phase IV cluster randomised clinical trial. BMC Fam Pract 2012;13:109.

28. Johnson $\mathrm{M}$, Jones $\mathrm{R}$, Freeman $\mathrm{C}$, et al. Can diabetes prevention programmes be translated effectively into real-world settings and still deliver improved outcomes? A synthesis of evidence. Diabet Med 2013;30:3-15.

29. Balion CM, Raina PS, Gerstein HC, et al. Reproducibility of impaired glucose tolerance (IGT) and impaired fasting glucose (IFG) classification: a systematic review. Clin Chem Lab Med 2007;45:1180-5.

30. Bartoli E, Fra GP, Carnevale Schianca GP. The oral glucose tolerance test (OGTT) revisited. Eur J Intern Med 2011;22:8-12. 